\title{
CONTRIBUIÇÕES DAS OFICINAS TERAPÊUTICAS E EDUCATIVAS NOS CENTROS DE ATENÇÃO PSICOSSOCIAL INFANTOJUVENIL: REVISÃO INTEGRATIVA
}

\author{
Simone Cambraia Machado Haygert ${ }^{1}$; Fernanda Figueira Marquezan ${ }^{2}$; Diego Carlos
} Zanella $^{3}$

\section{RESUMO}

Os Centros de Atenção Psicossocial Infantil (CAPSi) surgem como uma alternativa de cuidado na saúde mental de crianças e adolescentes. As oficinas terapêuticas são atividades grupais destinadas à socialização familiar e social dos usuários, à expressão de sentimentos e emoções, ao desenvolvimento de habilidades e da autonomia e ao exercício da cidadania. Os conceitos de oficina educativa e oficina terapêutica possuem uma intersecção e são dispositivos grupais de aprendizagem. O objetivo deste estudo foi realizar uma revisão integrativa de literatura sobre as oficinas terapêuticas desenvolvidas nos CAPSi. Foram selecionados três artigos para leitura integral que abordaram oficinas de rádio, confecção de bonecos e percepções de familiares de adolescentes acerca das oficinas terapêuticas no CAPSi. Conclui-se, a partir deste trabalho, o caráter educativo existente nas oficinas terapêuticas, visto que ações temáticas nos CAPS potencializam a interação social, melhoram a aprendizagem de novas formas de se comunicar e promovem o autoconhecimento.

Palavras-chave: Atividades grupais; Interação psicossocial; Saúde mental.

Eixo Temático: Ciências humanas.

\section{INTRODUÇÃO}

Historicamente, no Brasil, a saúde mental de crianças e adolescentes é diretamente associada com a responsabilidade de setores educacionais e assistenciais. A família apresenta-se como o primeiro meio de cuidado integral às

\footnotetext{
${ }^{1}$ Mestranda em Ensino de Humanidades e Linguagens - Universidade Franciscana (UFN) - e-mail: s.cambraia@ufn.edu.br

2 Professora do Mestrado em Ensino de Humanidades e Linguagens, da Universidade Franciscana (UFN) - e-mail: marquezanfernanda@gmail.com

3 Professor e coordenador do Mestrado em Ensino de Humanidades e Linguagens, da Universidade Franciscana (UFN) - e-mail: diego.zanella@ufn.edu.br
} 
crianças e aos jovens, precedida pela escola e permeado pelo meio social e pela saúde pública, que em nosso país é referenciada ao Sistema único de Saúde (SUS). A partir do final da década de 70 , inicia-se uma mobilização para que ocorra a reforma psiquiátrica brasileira, a qual tem sua lei aprovada e sancionada em 2001 Lei Federal № 10.216 (BRASIL, 2004). Nesse sentido, os Centros de Atendimento Psicossocial Infantil (CAPSi) surgem como um dispositivo de assistência especializada, os quais têm como um de seus objetivos a reinserção social a partir da integração de ações de saúde, educação e assistência social.

Dentre as estratégias de tratamento oferecidas no CAPSi estão as Oficinas Terapêuticas, as quais, segundo o Ministério da Saúde (BRASIL, 2004), são caracterizadas como atividades grupais destinadas à socialização familiar e social dos usuários, à expressão de sentimentos e emoções, ao desenvolvimento das habilidades e da autonomia e ao exercício da cidadania. Assim, as oficinas representam uma importante ferramenta de autopercepção e olhar sobre o outro, levando, com isso, a uma ressocialização. Nesta lógica de pensar coletivo, faz-se presente o respeito à diversidade, e o estímulo à capacidade criadora de cada sujeito envolvido (AZEVEDO; MIRANDA, 2011).

As oficinas oportunizam um diálogo com a arte, a educação e a saúde, podendo desenvolver potenciais criativos, artísticos e socioculturais. Entre as suas modalidades, existem as oficinas educativas, as quais, para Candau e Zenaide (1999), são consideradas:

[...] espaços de construção coletiva de um saber, de análise da realidade, de confrontação e intercâmbio de experiências, de exercício concreto dos direitos humanos. A atividade, a participação, a socialização da palavra, a vivência de situações concretas através de sócio-drama, a análise de acontecimentos, a leitura e discussão de textos, a realização de vídeosdebate, o trabalho com diferentes expressões da cultura popular, etc., são elementos presentes na dinâmica das oficinas (p. 24).

A partir dessa construção, as atividades desenvolvidas nas oficinas tornam-se momentos terapêuticos que permitem diferentes abordagens sobre as vivências dos participantes. Para Galletti (2004), o dispositivo de oficina é, geralmente, descrito quando se refere a novas propostas terapêuticas, e não se caracterizam como modelo hegemônico de intervenção, e sim como uma gama diversa de formas, 
processos e linguagens. Nesse contexto, disponibiliza-se ao sujeito envolvido o papel de se assumir como autor de sua história de vida e como aprendiz ao escutar a história dos outros, podendo, com isso, agir como agente transformador de si e do seu contexto, aspirando novas perspectivas pessoais e sociais (GRACIANI, 2005).

Diante de tais considerações, o presente estudo busca relacionar a oficina terapêutica e a oficina educativa, encontrar suas familiaridades e apresentar abordagens que possam aproximá-las e entrelaçá-las. Dessa forma, o objetivo deste artigo é apresentar uma revisão integrativa de literatura sobre as oficinas terapêuticas desenvolvidas nos CAPSi, para identificar possíveis abordagens educativas em um espaço não formal de educação.

Os resultados indicam que há uma intersecção entre o conceito de oficina educativa e oficina terapêutica enquanto dispositivos grupais de aprendizagem. A forma como essa relação acontece é ímpar e aponta para novas configurações na prática em saúde mental.

\section{MATERIAIS E MÉTODOS}

O artigo é uma revisão integrativa de literatura, método que possibilita a síntese e análise do que existe de produção sobre determinado fenômeno (BROOME, 2006). O termo 'integrativa' tem origem na integração de opiniões, conceitos ou ideias provenientes das pesquisas utilizadas no método (WHITEMORE; KNAFL 2005). Assim, em um estudo que se busca construir um conhecimento sobre as possíveis inter-relações entre as oficinas terapêuticas e educativas, tornase necessário integrar os assuntos. Dessa forma, trata-se de um método específico que resume o passado da literatura empírica ou teórica, para fornecer uma compreensão mais abrangente de um fenômeno particular (BROOME, 2006).

Empregaram-se como base de dados digitais as seguintes plataformas: Scientific Eletronic Library Online (SciELO) (https://www.scielo.br/), Pubmed (https://pubmed.ncbi.nlm.nih.gov) e Google Scholar (https://scholar.google.com.br/). Foi realizada uma estratégia de busca com os seguintes descritores em português: "Saúde Mental"; "Arte"; "Oficina Terapêutica" e "Centro de Atenção Psicossocial infantil", os quais foram combinados entre si com a utilização do operador booleano 
"AND". As combinações utilizadas foram as seguintes: Centro de Atenção Psicossocial Infantil AND saúde mental AND arte Oficina Terapêutica. Após a combinação foram então lidos os títulos para a primeira forma de seleção dos estudos.

Quadro 1 - Artigos pesquisados

\begin{tabular}{|l|l|l|l|l|}
\hline Plataforma & $\begin{array}{l}\text { Artigos } \\
\text { Encontrados }\end{array}$ & Incluídos & Excluídos & $\begin{array}{l}\text { Total para } \\
\text { análise }\end{array}$ \\
\hline Pubmed & 1 & 0 & ${ }^{*}$ CAPS AD & 0 \\
\hline Scielo & 2 & 1 & ${ }^{* 1}$ CAPS AD & 1 \\
\hline Google & 60 & 44 & $\begin{array}{l}{ }^{7} \text { artigos duplicados, } \\
\text { outros CAPS } \\
9 \quad \begin{array}{l}\text { Teses } \\
\text { dissertações }\end{array}\end{array}$ & \\
\hline Total & & & 45 artigos para análise inicial \\
\hline
\end{tabular}

Fonte: Os autores (2021).

Como critérios de inclusão, adotaram-se artigos sobre oficinas terapêuticas desenvolvidas nos CAPSi, publicados entre os anos de 2001 e 2021, com texto completo, com acesso gratuito e na língua portuguesa. Foram excluídas teses e dissertações, revisões de literatura, artigos que foram desenvolvidos em CAPS álcool e drogas e/ou CAPS adulto e artigos duplicados.

Desse modo, foram selecionados para análise inicial 45 artigos. Após a leitura dos títulos e dos resumos, foram selecionados três artigos, os quais se adequavam aos critérios de inclusão já citados anteriormente para compor essa revisão, todos associados com a prática de oficinas educativas, sendo apresentados no Quadro 2.

\section{RESULTADOS E DISCUSSÕES}

A seguir apresenta-se uma análise e intepretação dos textos selecionados, contextualizando com a literatura sobre as temáticas oficinas terapêuticas e oficinas educativas. 


\section{Quadro 2 - Artigos selecionados}

\begin{tabular}{|c|c|c|c|c|}
\hline Autor/Ano & Título & Objetivo & Metodologia & Conclusão \\
\hline $\begin{array}{l}\text { Souza, E.F.C.; } \\
\text { Dacal, C.S; } \\
\text { Souto,T.S.; } \\
\text { Soares, R.S., } \\
\text { Sant'Anna,V. } \\
\text { N.(2016) }\end{array}$ & $\begin{array}{l}\text { Quem é o dono } \\
\text { da voz? Uma } \\
\text { experimentação } \\
\text { radiofônica em } \\
\text { um centro de } \\
\text { atenção } \\
\text { psicossocial } \\
\text { infantil }\end{array}$ & $\begin{array}{l}\text { Trabalhar } \\
\text { vivência da } \\
\text { sexualidade no } \\
\text { cotidianor dos } \\
\text { adolescentes do } \\
\text { serviço, visando ao } \\
\text { desenvolvimento } \\
\text { da autonomia e do } \\
\text { protagonismo e à } \\
\text { redução de danos. }\end{array}$ & $\begin{array}{l}\text { Descreveram } \\
\text { o processo } \\
\text { de oficinas } \\
\text { radiofônicas } \\
\text { entre } \\
\text { setembro- } \\
\text { novembro de } \\
2015 .\end{array}$ & $\begin{array}{l}\text { Tratou-se de } \\
\text { dispositivo para dar } \\
\text { lugar à produção de } \\
\text { novas linguagens e à } \\
\text { possibilidade de nova } \\
\text { significação narrativas. } \\
\text { discursos e narrativas. } \\
\text { Pode-se, então, afirmar } \\
\text { que a voz ganhou seu } \\
\text { dono e assim hoje } \\
\text { sabemos quem é o } \\
\text { dono da voz. }\end{array}$ \\
\hline \begin{tabular}{l}
\multicolumn{2}{l}{ Camargo, } \\
V.P., Lena, \\
M.S., Dias, \\
H.Z.J, Roso, \\
A.R.; et al., \\
$(2011)$
\end{tabular} & $\begin{array}{ll}\text { Costurando } & \\
\text { saúde: } & \\
\text { Possibilidades } & \text { de } \\
\text { integração } & \text { por } \\
\text { meio da } & \\
\text { confecção } & \text { de } \\
\text { bonecos(as) } & \text { de } \\
\text { pano em um } & \text { uns infantil } \\
\text { CAPS }\end{array}$ & $\begin{array}{ll}\text { Apresentar } & \text { os } \\
\text { resultados } & \\
\text { da atividade } & \text { de } \\
\text { confecção } & \text { de } \\
\text { bonecos(as). } & \end{array}$ & $\begin{array}{l}\text { Descreveram } \\
\text { o } \\
\text { desenvolvim } \\
\text { ento de } \\
\text { oficina } \\
\text { terapêutica } \\
\text { que fez uso } \\
\text { da técnica de } \\
\text { confecção de } \\
\text { bonecos no } \\
\text { período de } \\
\text { janeiro a } \\
\text { março de } \\
2009 \text {. }\end{array}$ & $\begin{array}{l}\text { A confecção dos(as) } \\
\text { bonecos(as) não } \\
\text { somente propiciou um } \\
\text { novo ensaio da } \\
\text { experiência transicional, } \\
\text { mas, acima de tudo, } \\
\text { favoreceu um espaço } \\
\text { sem estigmatização, no } \\
\text { qual os participantes } \\
\text { puderam reconhecer-se } \\
\text { como sujeitos criativos e } \\
\text { capazes de adquirir } \\
\text { competências } \\
\text { psicológicas e culturais. }\end{array}$ \\
\hline $\begin{array}{l}\text { Noronha, } \\
\text { A.A., Folle, D.; } \\
\text { Guimarães, } \\
\text { A.N., Brum, } \\
\text { M.L.B; } \\
\text { Schneider, } \\
\text { J.F.;Motta, } \\
\text { M.G.C.(2016) }\end{array}$ & $\begin{array}{ll}\text { Percepções } & \text { de } \\
\text { familiares } & \text { de } \\
\text { adolescentes } & \\
\text { sobre oficinas } \\
\text { terapêuticas em } \\
\text { um centro de } \\
\text { atenção } & \\
\text { psicossocial } & \\
\text { infantil } & \end{array}$ & $\begin{array}{ll}\text { Conhecer } & \text { as } \\
\text { percepções } & \text { de } \\
\text { familiares de } & \text { de } \\
\text { adolescentes sobre } \\
\text { oficinas } \\
\text { terapêuticas em um } \\
\text { Centro de Atenção } \\
\text { Psicossocial } \\
\text { Infantil. }\end{array}$ & $\begin{array}{l}\text { Descreveram } \\
\text { sobre as } \\
\text { oficinas com } \\
\text { familiares } \\
\text { e/ou } \\
\text { acompanhan } \\
\text { tes de } \\
\text { adolescentes } \\
\text { onde se } \\
\text { realizaram } \\
\text { entrevistas, } \\
\text { interpretadas } \\
\text { e analisadas } \\
\text { no ano de } \\
2013 \text {. }\end{array}$ & 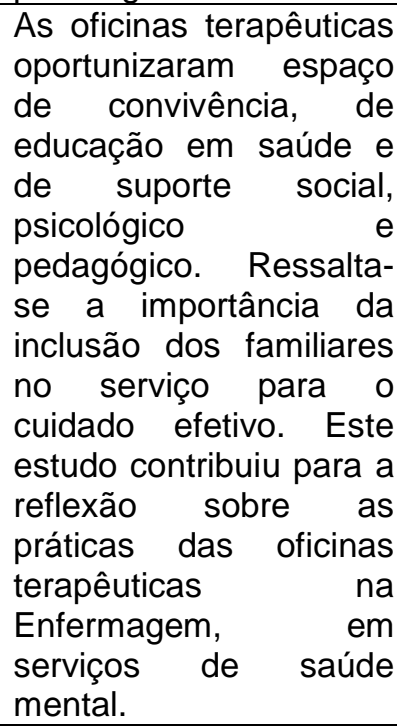 \\
\hline
\end{tabular}

Fonte: Os autores (2021).

No estudo de Souza, Dacal, Souto, Soares, Sant'Anna, (2016), a oficina terapêutica foi utilizada como um dispositivo que proporcionou aos adolescentes de uma comunidade de São Paulo uma experiência de comunicação por meio de uma 
linguagem radiofônica, desenvolvendo a temática sobre a sexualidade de adolescentes. Sabe-se o quanto esta fase da vida, marcada por transformações físicas; psico-cognitivas e sociais, necessita de uma atenção intrafamiliar, escolar e de serviços de saúde. Em uma revisão integrativa, Nascimento, Bernardes, Conceição, Silveira e Fajersztajn (2021) observaram uma escassez de informações sobre temas referentes a doenças sexualmente transmissíveis, gravidez, métodos contraceptivos e mudanças físicas direcionadas aos adolescentes. Assim como perceberam que existem mitos e tabus sobre a sexualidade tanto nas relações familiares como no entorno social.

Para Aberastury (2007), no período da adolescência, há modificações psicológicas e corporais em que o adolescente perde a sua condição de criança e passa a ter uma nova relação com seu corpo, sua sexualidade, com os seus pais e com o mundo. Essa falta de comunicação entre os locais com os adolescentes, dificulta até mesmo a aproximação deles com seus familiares, professores e outros adultos. Brandão (2004) afirma que, na atualidade, é impossível fugir do diálogo com argumentação sobre o tema da sexualidade na educação dos filhos. Ou seja, existe uma grande necessidade de se associar oficinas terapêuticas a práticas educativas que utilizem a temática da sexualidade como objeto de diálogo.

No artigo intitulado Costurando saúde: Possibilidades de integração por meio da confecção de bonecos(as) de pano em um CAPS infantil, de Camargo, Lena, Dias, Roso (2011), a temática da oficina terapêutica foi a confecção de bonecos. Isso proporcionou aos participantes uma relação de criação do boneco, e uma forma de expressar seus sentimentos internalizados. Além disso, também se proporcionou educação sobre a técnica da confecção, molde, recorte, costura e afins.

Os autores basearam-se na perspectiva psicanalítica winnicottiana, teoria que valoriza o brincar no processo terapêutico e salienta que durante o desenvolvimento emocional a criança passa por várias etapas, onde, muitas vezes, utiliza-se do objeto transicional como mediador nessa passagem de um estado para outro.

Nesta oficina, os bonecos, além de servirem como objeto/brinquedo, também remetem a este objeto transicional. Para as crianças, o brincar é sua forma de 
expressão e torna-se a sua forma de se relacionar com seus sentimentos e com o universo ao seu redor (WINNICOTT, 1975).

Quanto ao uso de arteterapia, esta pode ser utilizada como prática da arte enquanto processo criativo. Segundo a Associação Americana de Arteterapia AATA (2009) o fazer arte na arteterapia é referenciado como processo de autoexpressão que ajuda as pessoas a resolver conflitos, desenvolver habilidades, reduzir estresse, aumentar autoestima e autoconsciência, então, seu uso como intervenção terapêutica mostrasse extremamente desafiador de sentidos. Dessa forma, o confeccionar do boneco serve como um meio de expressão e interlocução no meio.

Nos CAPS, a arte, em suas várias expressões, vem sendo utilizada nas oficinas terapêuticas e serve como proposta de intervenção em que a prioridade é o processo do fazer - a experiência do vivido. Nesse contexto, a linguagem nem sempre é a da palavra. Winnicott (1975) salientou que, na escuta terapêutica, existe a delicadeza do que é pré-verbal, não verbalizado e não verbalizável.

O estudo dos autores Noronha, Folle, Guimarães, Brum, Schneider, Motta, (2016), no artigo Percepções de familiares de adolescentes sobre oficinas terapêuticas em um centro de atenção psicossocial infantil objetivou conhecer as percepções de familiares de adolescentes sobre oficinas terapêuticas oferecidas como plano terapêutico em um CAPSi. Como conclusão do estudo, os autores perceberam que as oficinas proporcionam a melhora da convivência social e familiar, a redução dos danos associados ao consumo de drogas, a estabilidade e a diminuição das crises. Porém, relatam que ainda há pouco conhecimento sobre o que as oficinas são, como ocorrem e qual a sua importância no tratamento das pessoas com transtornos mentais, para a promoção de uma assistência em saúde mental condizente com suas realidades de vida.

Nesse contexto, destaca-se a importância da intervenção terapêutica de oficinas com familiares e/ou cuidadores de adolescentes usuários, porque a família, enquanto instituição social, é de grande influência para o dinamismo do tratamento dos adolescentes em sofrimento psíquico. Schrank (2008) realizou um estudo de caso para identificar as ações de saúde mental desenvolvidas no CAPS voltadas 
para a família no cuidado do indivíduo com sofrimento psíquico. Relata, em seu estudo, que é necessário um trabalho em conjunto entre a equipe e a família, para que o cuidado seja integral. Dessa forma, estratégias como as oficinas têm como objetivos: orientar, esclarecer, facilitar, incentivar e apoiar a família nessa proposta de trabalho, objetivando, desse modo, a convivência e o respeito frente à experiência do sofrimento psíquico.

A proposta terapêutica do CAPS, como uma nova forma de cuidado da saúde mental, compreende uma série de atividades e dinâmicas diversificadas que devem ser pensadas e discutidas por todos os envolvidos no serviço, isto é, profissionais de nível técnico, de ensino superior, usuários e familiares. Assim, atividades, como as oficinas terapêuticas, privilegiam os modos de subjetividade de cada sujeito envolvido, como a valorização de oferecer um espaço de interação grupal.

Nos três artigos selecionados para esta revisão integrativa, as oficinas adequadas às demandas do serviço e compatíveis com as necessidades dos usuários, uma vez que o CAPSi é um serviço de atenção diária destinado ao atendimento de crianças e adolescentes gravemente comprometidos psiquicamente. Também promove acessibilidade de tratamento e busca ampliação do conhecimento clínico e epidemiológico (COUTO; DUARTE; DELGADO, 2008).

As oficinas, para Anastasious e Alves (2003), caracterizam-se como uma estratégia do fazer pedagógico, onde há um espaço de construção e reconstrução do conhecimento. Pode-se pensar, descobrir, reinventar, criar e recriar, o que pode contribuir muito para tratamentos com crianças e adolescentes que necessitam de uma abordagem mais acolhedora como ocorrem nos CAPSi.

Dessa forma, oficinas terapêuticas realizadas em um espaço não formal de educação, como os CAPSi, podem se tornar oficinas educativas. Uma vez que abordam temáticas necessárias a uma determinada população, utilizam dinâmicas similares que têm como principal objetivo a construção coletiva de um saber, de análise da realidade, de confrontação e de intercâmbio de experiências, de exercício concreto dos direitos humanos (CANDAU; ZENAIDE, 1999). 


\section{CONCLUSÃO}

O desenvolvimento de oficinas terapêuticas nos CAPS possibilita a reunião de grupos de usuários, familiares, cuidadores ou profissionais. Nesse contexto, há um caráter educativo nas oficinas terapêuticas, visto que ações temáticas, nos CAPS, potencializam a interação social e a aprendizagem de novas formas de se comunicar com o outro, promovendo autoconhecimento.

O primeiro artigo utiliza-se da linguagem radiofônica para discutir a sexualidade de adolescentes, o segundo usa a confecção de bonecos como arteterapia e trazem a visão psicanalítica winnicottiana, utilizando a brincadeira como uma forma de expressão. Por fim, o terceiro estudo busca compreender quais são as percepções dos familiares de adolescentes sobre oficinas terapêuticas.

Posto isso, os três artigos utilizados como referências para esse estudo possibilitaram os autores ampliarem seus conceitos sobre como as oficinas podem assumir papéis enquanto terapêuticas e educativas. Essas duas áreas, quando integradas ao Centro de Atenção Psicossocial infantil, contribuem para a proximidade dos usuários desse serviço, melhorando o vínculo entre os profissionais envolvidos, familiares e os usuários. Dito isto, integrar atividades de oficinas terapêuticas com abordagens educativas auxilia ainda mais para o cuidado da saúde mental.

\section{REFERÊNCIAS}

ABERASTURY, A. O adolescente e a liberdade. In: ABERASTURY, A.; KNOBEL, M. (Org.). Adolescência normal: um enfoque psicanalítico. Porto Alegre-RS: Artmed, 2007. (Original de 1981). p. 13-23.

ANASTASIOU, L. G. C; ALVES, L. P. Processo de ensinagem na universidade: pressupostos para as estratégias de trabalho em aula. Joinville-SC: Univille, 2003.

AZEVEDO, D. M., MIRANDA, F. A. N. Oficinas terapêuticas como instrumento de reabilitação psicossocial: percepção de familiares. Escola Anna Nery, v. 15, n. 2, p. 
339-345, abr./jun. 2011. Disponível em: http://www.scielo.br/pdf/ean/v15n2/v15n2a 17. Acesso em: 26 jul. 2021.

BRANDÃO, E. R. Iniciação sexual e afetiva: exercício da autonomia juvenil. In: HEILBORN, M. L. (Org.). Família e sexualidade. Rio de Janeiro-RJ: Editora FGV, 2004. p. 63-86.

BRASIL Ministério da Saúde. Secretaria Executiva. Secretaria de Atenção à Saúde. Legislação em saúde mental: 1990-2004. 5a ed. ampl. Brasília-DF: Ministério da Saúde; 2004.

BROOME, M. E. Integrative literature reviews for the development of concepts. In: RODGERS, B. L.; CASTRO, A. A. Revisão sistemática e meta-análise. 2006. Disponível em: http://www.metodologia.org/meta1.PDF. Acesso em: 12 jan. 2011.

Camargo, V.P., Lena, M.S., Dias, H.Z.J, Roso, A.R.; Costurando saúde: possibilidades de integração por meio da confecção de bonecos(as) de pano em um CAPS infantil. Psicologia Argumento, v. 29, n. 64, p.101-108, jan./mar. 2011.

CANDAU, V. M., ZENAIDE, M. N. Educação em Direitos Humanos: Uma proposta de trabalho, 1999. Disponível em: http://www.dhnet.org.br/direitos/militantes/veracan dau/candau_edh_proposta_trabalho.pdf. Acesso em: 26 jul. 2021.

COUTO, M. C. V.; DUARTE, C. S.; DELGADO, P. G. G. A saúde mental infantil na Saúde Pública brasileira: situação atual e desafios. Revista Brasileira de Psiquiatria, v. 30, p. 390-398, 2008.

GALLETTI, M. C. Oficinas em saúde mental: instrumento terapêutico ou intercessor clínico? Goiânia-GO: Editora da UCG, 2004. 146 p. 
GRACIANI, M. S. S. Pedagogia social de rua: análise e sistematização de uma experiência vivida. 5. ed. São Paulo-SP: Cortez; Instituto Paulo Freire, 2005. Coleção Prospectiva.

Nascimento J.C.M, Bernardes, L. M., Conceição, L.M., Silveira, O.S. e Fajersztajn, L. Educação em Saúde sobre sexualidade na adolescência: uma revisão integrativa da literatura. Leopoldianum, v.47, n. 131, 2021.

Noronha, A.A., Folle, D.; Guimarães, A.N., Brum, M.L.B; Schneider, J.F.;Motta, M.G.C.(2016). Percepções de familiares de adolescentes sobre oficinas terapêuticas em um centro de atenção psicossocial infantil. Revista Gaúcha de Enfermagem, v. 37, n. 4, p. e56061, dez. 2016. DOI: 10.1590/19831447.2016.04.56061.

SCHRANK, G.; OLSCHOWSKY, A. O centro de Atenção Psicossocial e as estratégias para inserção da família. Revista da Escola de Enfermagem da USP [online], v. 42, n. 1, p. 127-134, 2008.

SOUZA, E. F. C. Souza, E.F.C.; Dacal, C.S; Souto,T.S.; Soares, R.S., Sant'Anna,V.N. Quem é o dono da voz? Uma experimentação radiofônica em um Centro se Atenção Psicossocial Infantil. Cadernos Brasileiros de Saúde Mental, Florianópolis, v. 8, n. 18, p. 81-95, 2016.

WHITTEMORE, R.; KNAFL, K. The integrative review: updated methodology. Journal of Advanced Nursing, Oxford, v. 52, n. 5, p. 546-553, dez. 2005.

WINNICOTT, D. W. O Brincar e a Realidade. Rio de Janeiro-RJ: Imago ed., 1975. 